\title{
Appropriation effects in the estimation of modal damping
}

\author{
Mohamed Krifa, Noureddine Bouhaddi, Scott Cogan \\ FEMTO-ST Institute, Department of Applied Mechanics, University of Franche-Comté \\ UBFC, 24, Rue de l'Epitaphe, F-25000 Besancon, France \\ mohamed.krifa@femto-st.fr,noureddine.bouhaddi@femto-st.f, scott.cogan@femto-st.fr
}

\begin{abstract}
Damping presents one of the most important physical aspects to model and estimate, since it plays a large role in determining the performance of a dynamic system and the amplitude of vibrations. The present study employs the modal strain energy method to estimate the modal damping associated with the localized dissipative interfaces of a global linear structure. This method is accurate in the case of proportional or classical damping model. But in the real case when modes are coupled with damping due to the localization of the dissipation, as in the case of most assembled structures, this method may present significant errors. In this paper an appropriation method is proposed and associated to the modal strain energy method in order to get a good estimation of the modal damping. The impact of appropriation on the modal damping estimation in the case of non-proportional viscous damping model is studied for a multi-degree of freedom system. Results are compared with the reference one obtained by the state space method. Simulated academic examples, where accurate estimations of the exact solutions are available, will be used to illustrate the methodology and to explore the potential difficulties that may arise in more complex industrial applications.
\end{abstract}

Keywords: appropriation method, non-proportional damping, modal strain energy method, bolted joints, localized dissipation. 


\section{Introduction}

Analytical techniques for estimating structural vibration have become increasingly sophisticated. However the estimation of damping remains difficult and uncertain, due to the variety and complexity of its physical origin. Indeed, damping can arise from many different sources, such as (Mead 1998): intrinsic damping of the structural material, coulomb friction and partial-impact energy loss at structural interfaces (Gaul and al 2008, Caignot and al 2010), energy lost into surrounding air, water or ground and into contained fluids; friction between the structure and mounted equipment, furnishings, payload and people, and viscous damping (Adhikari 2014, Krifa and al 2015) between sliding and lubricated machines surfaces.

In all generality, the dissipated forces can be expressed as a non-linear function of the displacement $\mathrm{y}$ and the velocities \&.

$f_{d}=f_{d}(y, x)$

For simplification reasons, in this study the damping is assumed to be linear with respect to the velocities (viscous damping) and independent of $y$.

The main purpose of this paper is to highlight the performance of appropriation method for the estimation of modal damping in the case of localized dissipation modeled with viscous damping model. Advantages and drawbacks of the modal strain method with and without appropriation will be presented and compared to the reference one obtained by the state space method, in order to explore the potential difficulties that may arise in more complex industrial applications.

\section{Theoretical background}

In order to estimate the damping of structures with a multi-degree of freedom when the modes are well separated, we can use the following methods: the state space method, modal strain energy and the appropriation method. We describe here briefly these methods.

Assuming a linear and dissipative problem of assembled structures, the discrete form of the damped vibration problem may be governed by the following equation:

$$
M(t)+C y(t)+K y(t)=f(t)
$$

where $\mathrm{K}, \mathrm{M}$ and $\mathrm{C}$ are respectively the stiffness, mass and damping matrices, $\mathrm{y}$ is the response of the system, $\mathrm{f}$ is the vector of the external loads. We distinguish two cases : proportional damping (Rayleigh 1896) and non-proportional damping. In both cases the frequency response of the system governed by equation (2) is equal to 
$y(\omega)=\left(K+j \omega C-\omega^{2} M\right)^{-1} \times f$

Unfortunately, the use of equation (3) may be computationally very expensive especially for large order systems. The assumption of proportional damping is generally used in order to resolve the system with an acceptable cost and duration by projecting on the undamped modal basis :

$y\left(\omega_{v}\right)=\sum_{i=1}^{n} \phi_{i} q_{i}$

where $\phi_{\mathrm{i}}$ and $\mathrm{q}_{\mathrm{i}}$ are respectively the eigenmode and the modal amplitude corresponding to the $i^{\text {th }}$ eigenfrequency $\omega_{i}$. The contribution of all modes is taken into account using this equation. But the contribution of all these modes can contribute to a non accurate estimation of the modal damping of a specific mode as demonstrated later in the numerical simulations.

To avoid this problem, a projection of the response on a single mode of vibration is generally used, thus ensuring an accurate estimation of the modal damping, so the equation (4) becomes

$y\left(\omega_{v}\right)=\phi_{v} q_{v}$

\subsection{Reference method}

The reference method is the state space method (Geradin and Rixen 2014). It is chosen for two main reasons: first for its accuracy in computing the modal damping, and secondly for its capacity to be used in both non-proportional (localized) and proportional damping cases. The state space equation is classically written in the following form:

$\left[\begin{array}{cc}C & M \\ M & 0\end{array}\right]\left[\begin{array}{l}\dot{y}(t) \\ \ddot{y}(t)\end{array}\right]=\left[\begin{array}{cc}-K & 0 \\ 0 & M\end{array}\right]\left[\begin{array}{l}y(t) \\ \dot{y}(t)\end{array}\right]$

However, the state space method uses complex eigenvalue solutions by solving a double size system $(2 n \times 2 n)$. So, this method is time-consuming for large finite element model. And there is no physical meaning behind the use of complex variables.

The resolution of the equation (6) gives $n$ complex eigenvalues $s_{v}$ and $n$ conjugate complex eigenvalues $\bar{s}_{v}$. When the modal damping factor $\xi_{v}$ verifies $\xi_{v}=1$, these eigenvalues can be expressed as follows:

$s_{v}=-\xi_{v} \omega_{v} \pm j \omega_{v} \sqrt{1-\xi_{v}^{2}}$ 
Eigenfrequency and modal damping are deduced from the equation (7) by the following expressions :

$$
\begin{aligned}
& \omega_{v}=\sqrt{\operatorname{Re}^{2}\left(s_{v}\right)+\operatorname{Im}^{2}\left(s_{v}\right)} \\
& \xi_{v}=-\frac{\operatorname{Re}\left(s_{v}\right)}{\omega_{v}}
\end{aligned}
$$

This method has been implemented in Matlab and will be considered as the reference one, as it generates "the exact" estimation of the modal damping.

\subsection{Modal strain method (MSE)}

The Modal Strain Energy method (MSE) was firstly suggested by Ungar and Kerwin in 1962, and has been used since to address viscoelastic damping problems of sandwich structures by Johnson and Klenholz (1982). Later, a modified MSE was proposed by (Dokainish 1995) in order to improve the estimation of modal damping. The advantage of the MSE method is that it allows one to compute modal damping by a real instead of a complex, eigenvalue solution. Consequently, the computational cost is greatly reduced. The objective of the modal strain energy is to determine the damping factor corresponding to each vibration mode of the structure. It is based on the concept of the dissipated energy in the interfaces for which the close form expression of the loss factor is the ratio between dissipated energy and maximal potential energy, over a cycle of periodic vibration (Krifa and al 2015), as shown in this relation:

$\xi_{v}=\frac{1}{4 \pi} \frac{E_{v}^{d i s s}}{E_{v}^{p o t}}$

where $E_{v}^{\text {diss }}$ and $E_{v}^{\text {pot }}$ are respectively the dissipated energy and maximal potential energy.

Dissipated energy is calculated by the following expression:

$E_{v}^{d i s s}=\int_{0}^{\tau} \dot{y}(t)^{T} f_{d}(t) d t$

where

$-\tau=\frac{2 \pi}{\omega_{v}}$ cycle of periodic vibration of mode $v$.

- $f_{d}(t)=C \dot{y}(t)$ dissipative force

- $\dot{y}(t)=\operatorname{Re}\left(j \omega_{v} y\left(\omega_{v}\right) e^{j \omega_{v} t}\right)$ velocity 
The potential energy is calculated as follows:

$E_{v}^{p o t}=\frac{1}{2} y\left(\omega_{v}\right)^{T} K y\left(\omega_{v}\right)$

Equation (5) supposes that only one mode, in the same time, is responsible for the vibration, and that the others neighbouring modes are not involved when a forced harmonic excitation is applied. This is of course true only if an appropriate force is applied.

\section{Proposed appropriation method}

The force appropriation method is widely used in the aeronautical community to perform modal testing (Ewins 1995). The specificity of force appropriation testing is its ability to identify one by one the normal modes of vibration of the associated undamped system through the cancellation of the damping forces by the excitation system (Piranda 2001).

From the equation (2) one can deduce the frequency expression for a given angular frequency $\omega$.

$$
\left(K+j \omega C-\omega^{2} M\right) y(\omega)=f
$$

The appropriated force is then deduced from the equation (13) when the structure is oscillated at the resonance frequency $\omega_{v}$ :

$f_{v}=\left(K+j \omega_{v} C-\omega_{v}^{2} M\right) \phi_{v}=j \omega_{v} C \phi_{v}$

In the case of a complex structure where the physical damping matrix $\mathrm{C}$ is unknown, it is sufficient to apply a force proportional to the eigenmode in the following form:

$f_{v}=\alpha_{v} \times \phi_{v}$ where $\alpha_{v}=$ cte

Later we will discuss examples to show the effectiveness of this choice of appropriation in calculating the coefficients of modal damping by modal strain energy. 


\section{Numerical simulations}

The results of an academic example consisting of a system with 8 dofs masses, springs, and dampers located (between dofs 1 and 2, 4 and 5, 7 and 8) will be presented in this part (Figure 1). We show here the influence of extra-diagonal damping coefficients on the dynamic responses of the damped system, using the proposed method.

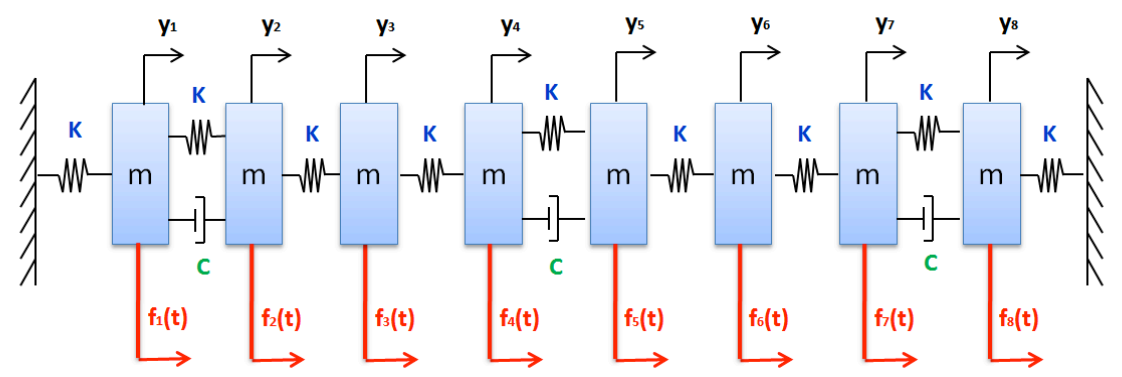

Fig. 1 : Linear array of $n$ spring-mass oscillators, $n=8, m=10 \mathrm{Kg}, \mathrm{k}=10^{5} \mathrm{~N} / \mathrm{m}$. Localized dampers are between $1^{\text {st }}$ and $2^{\text {nd }}, 4^{\text {th }}$ and $5^{\text {th }}, 7^{\text {th }}$ and $8^{\text {th }}$ masses, $c=50 \mathrm{Ns} / \mathrm{m}$.

The natural frequencies of the undamped system are summarised in the table 1 .

Table 1 : Eignfrequencies of the undamped system

\begin{tabular}{c|c|c|c|c|c|c|c|c}
\hline Mode & 1 & 2 & 3 & 4 & 5 & 6 & 7 & 8 \\
\hline $\begin{array}{c}\text { Eigen fre- } \\
\text { quency (Hz) }\end{array}$ & 5.53 & 10.89 & 15.92 & 20.46 & 24.38 & 27.57 & 29.91 & 31.35 \\
\hline
\end{tabular}

In the case of localized dissipation, the modal damping estimated by modal strain energy depends on the applied excitation. In the following, we will compare the influence of the type of the external force on the estimation of the modal damping.

If one applies a non-appropriated force to the localized spring mass damper system, the MSE method may not provide a good estimation of damping for all modes. For instance, the first excitation (a) applied gives a reasonable estimation for just the first five modes about $0.25 \%$ (Table $2 \mathrm{a}$ ), while the two coefficients $\xi_{6}$ and $\xi_{8}$ are miscalculated. One can notice an error equals to $13.98 \%$ and 18.91 $\%$ respectively for modes 6 and 8 , which is not acceptable.

Figure 2a shows a comparison of the coefficients of damping estimated between the reference method and the modal strain energy without force appropriation. The corresponding errors committed are given in Figure $2 b$. 

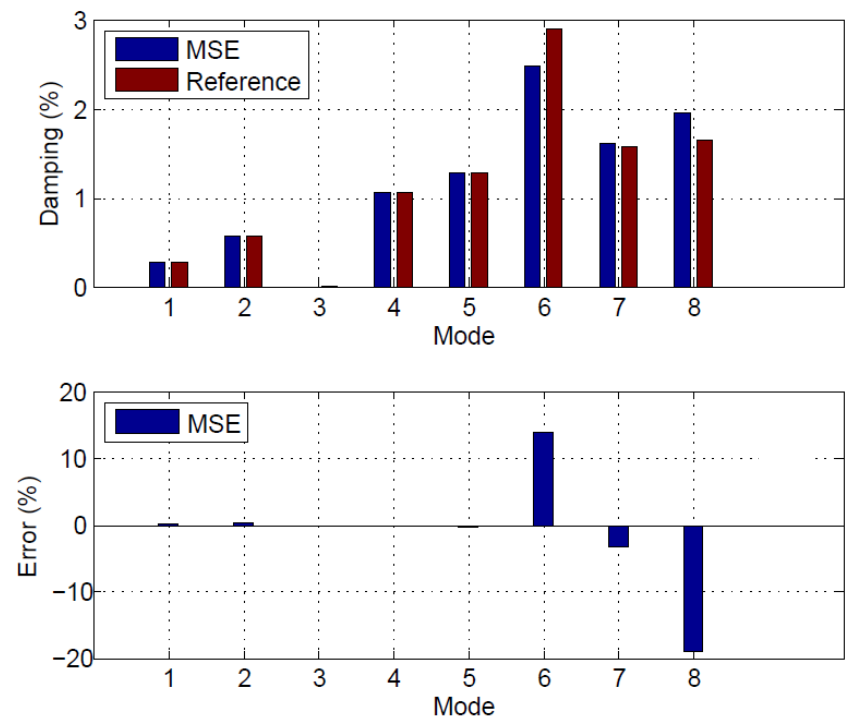

Fig. 2 : (a) : Comparison of estimated damping coefficients (b): Estimation Error

The errors of estimation can be explained by the importance of non-diagonal terms in the generalized damping matrix. In order to solve this problem of coupled modes by damping, one can apply an appropriated force using equation (14). Consequently, a good estimation of the modal damping by the appropriate force method is ensured.

Table 2: Impact of the appropriation force on the modal damping estimation. Three studied cases: (a) non appropriated force, (b) appropriated force, and (c) quasi-appropriated force

\begin{tabular}{|c|c|c|c|c|c|c|c|c|}
\hline & \multicolumn{8}{|c|}{ (a) Excitation $f_{v}=\left\{\begin{array}{llllllll}1 & 0 & 0 & 0 & 0 & 0 & 0 & 0\end{array}\right\}^{T}$} \\
\hline Mode & 1 & 2 & 3 & 4 & 5 & 6 & 7 & 8 \\
\hline$\xi_{v}(\%)$ & 0.29 & 0.57 & 0 & 1.07 & 1.28 & 2.48 & 1.62 & 1.95 \\
\hline \multirow[t]{2}{*}{$\operatorname{err}(\%)$} & 0.04 & 0.25 & 0 & -0.04 & -0.21 & 13.98 & -3.12 & -18.91 \\
\hline & \multicolumn{8}{|c|}{ (b) Appropriated Excitation $f_{v}=\omega_{v} C \phi_{v}$} \\
\hline$\xi_{v}(\%)$ & 0.29 & 0.57 & 0 & 1.07 & 1.27 & 2.88 & 1.57 & 1.64 \\
\hline \multirow[t]{2}{*}{$\operatorname{err}(\%)$} & -0.01 & -0.06 & 0 & 0.03 & -0.07 & 0 & 0.08 & 0.02 \\
\hline & \multicolumn{8}{|c|}{ (c) Quasi-appropriated Excitation $f_{v}=\alpha \phi_{v}$} \\
\hline$\xi_{v}(\%)$ & 0.29 & 0.57 & 0 & 1.07 & 1.26 & 2.88 & 1.56 & 1.64 \\
\hline $\operatorname{err}(\%)$ & -0.01 & 0.07 & 0 & 0.26 & 1.05 & 0 & 0.86 & 0.17 \\
\hline
\end{tabular}


For each eigenfrequency, the simulation with an appropriated excitation is run and the corresponding modal coefficient is obtained using the appropriate response. The advantage of this method is that appropriation guarantees the excitation of only one mode at a given time, so thereafter a good estimation of the modal damping is obtained (Table $2 b$ ).

However, the drawback of the appropriation method is that the appropriated force is expressed in terms of the damping matrix $\mathrm{C}$ which is not necessarily known in practical problems. To overcome this difficulty it is proposed to apply a quasi-appropriate force which is proportional to the eigenvector as expressed by the equation (15).

As illustrated in the Table $2 \mathrm{c}$, this quasi-appropriate force gives a good prediction of modal damping.

Figure 3 shows the frequency response using the modal damping estimated by the modal strain energy approach associated to the appropriation force method. The estimated FRF coincides with the reference FRF obtained using the reference state space method.
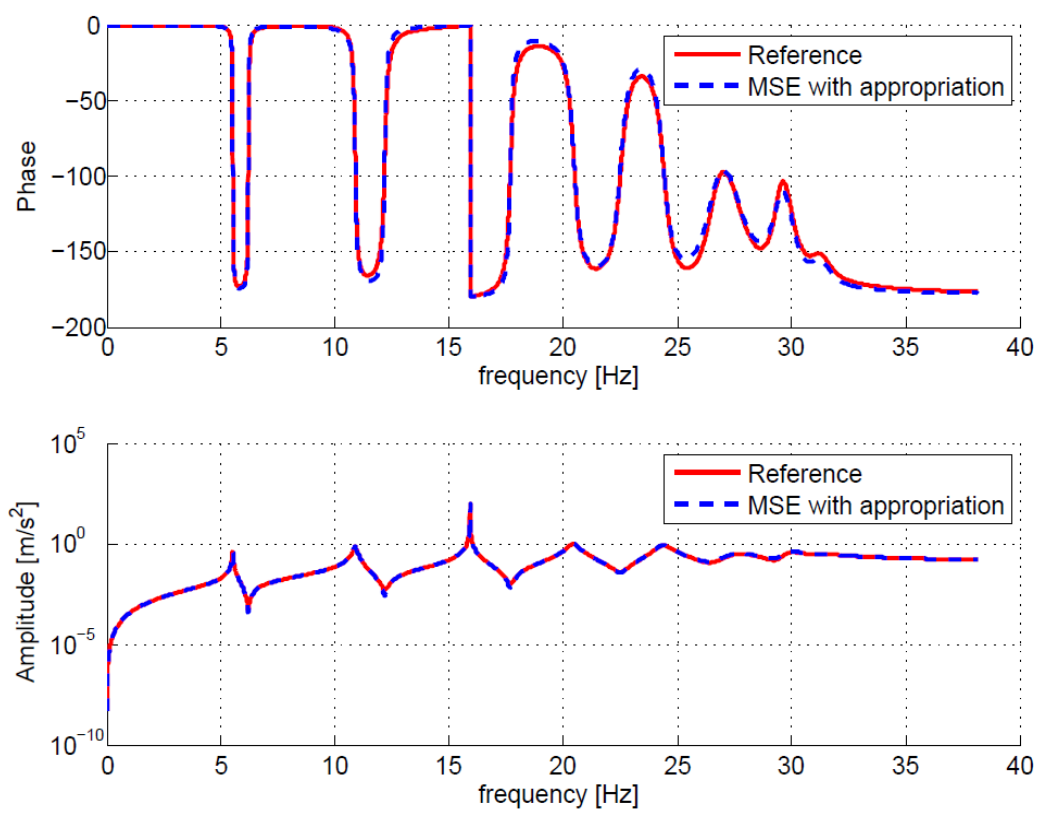

Fig. 3 : Forced response related to the non-proportional case using modal damping estimated by the proposed method 


\section{Conclusion}

The modal strain energy (MSE) method has been presented to estimate the modal loss factor of structures with viscous dampers. An appropriation approach has been proposed and associated to the MSE method. This method estimates the modal loss factor of structures with respect or not to the condition of proportionality. The results obtained by different methods (reference state space method and MSE method) are generally consistent with respect to the prediction of the modal loss factor, particularly when the structure has a proportional matrix damping. However, for a non-proportional matrix damping, both methods can give different results for certain excitations. This problem was address by using an appropriated excitation. Finally the modal strain energy associated with the appropriation method was illustrated by a 8 DOF spring-mass system with (proportional and non-proportional matrix damping).

\section{Acknowledgements}

Authors are grateful to the National Research Agency for their financial support of this project (contract ANR-12-MONU-00016-01).

\section{References}

Adhikari, S. (2013). Structural Dynamic Analysis with Generalized Damping Models: Analysis. John Wiley \& Sons.

Bograd, S., Reuss, P., Schmidt, A., Gaul, L., \& Mayer, M. (2011). Modeling the dynamics of mechanical joints. Mechanical Systems and Signal Processing, 25(8), 2801-2826.

Caignot A., Ladeveze P., Neron D. and Durand J.-F., Virtual testing for the prediction of damping in joints. Engineering Computations, 5, 621-644.15, 2010.

Dokainish, M. A., \& Mansour, W. M. (1995). A Modified MSE Method for Viscoelastic Systems: A Weighted Stiffness Matrix Approach. Journal of Vibration and Acoustics, 1001, 227.

Ewins, D. J. (1995). Modal testing: theory and practice (Vol. 6). Letchworth: Research studies press. 
Géradin, M., \& Rixen, D. J. (2014). Mechanical vibrations: theory and application to structural dynamics. John Wiley \& Sons..

Johnson, C. D., \& Kienholz, D. A. (1982). Finite element prediction of damping in structures with constrained viscoelastic layers. AIAA Journal, 20(9), 12841290.

Krifa, M., Bouhaddi, N., \& Cogan, S. (2015). Estimation of Modal Damping for Structures with Localized Dissipation. In Special Topics in Structural Dynamics, Volume 6 (pp. 179-191). Springer International Publishing.

Mead, D. J. (1999). Passive vibration control. John Wiley \& Sons Inc.

Piranda, J. (2001). Analyse modale expérimentale. Techniques de l'ingénieur,6180, 1-29.

Rayleigh, J. W. S. B. (1896). The theory of sound (Vol. 2). Macmillan.

Ungar, E. E., \& Kerwin Jr, E. M. (1962). Loss factors of viscoelastic systems in terms of energy concepts. The Journal of the acoustical Society of America,34(7), 954-957. 